\title{
Design and operation of a protection system for transformers with superconducting windings
}

\author{
F. van Overbeeke, K Oordt and LJ.M. van de Klundert
}

Twente University of Technology, Department of Applied Physics, PO Box 217. 7500 AE Enschede, The Netherlands

\begin{abstract}
Received 18 March 1985; revised 1 August 1985
Power transformers with superconducting windings need a protection system to prevent damage to the low-loss superconducting winding by an abnormally high current. The generally accepted protection technique which uses auxiliary coils has been analysed using a network representation. The current distribution between main and auxiliary coil is expressed in terms of geometrical parameters. Experimental data on current transfer and main coil recovery in a test transformer are presented and a method of obtaining a very low auxiliary coil current is suggested.
\end{abstract}

Keywords: superconductivity; transformers; design and operation; protection system

The idea of applying superconductors for the windings of large power transformers has existed since the discovery of type II superconductivity. However, the idea has always been rejected because of the unreliable quality of cryogenic apparatus and especially the unavoidable a.c. losses in superconductors. It was not until the beginning of the 1980s that the technology in both fields had been sufficiently developed to make this large-scale application of superconductivity economically feasible ${ }^{1}$. Only the parameters of the superconductor to be used have been defined; no wire is at present available that meets the very strict demands. However, a suitable wire has recently been developed on a laboratory $\operatorname{scale}^{2,3}$ and its commercial availability can be expected in the near future.

Because a.c. losses in superconductors are an increasing function of the volume of superconducting material, it is important not to use a greater mass than is strictly necessary for safe operation under normal conditions. Under abnormal conditions, e.g. in short-circuits, currents of 10-20 times the nominal value are possible.

The way generally proposed to deal with these fault currents is the use of an extra set of so-called auxiliary windings, made of normal conductors or well-stabilized and largely oversized superconductors, located around the low-loss main windings in such a way that under normal conditions a negligible current flows in these auxiliary windings. Figure 1 illustrates this concept in its various forms. Its principle was suggested by Jones in $1964^{4}$. He envisaged a composite consisting of a normal conductive layer supporting the superconductor, with the normal conductor located in the field-free region (Figure la).

However, this approach required that either each coil be made of only one layer of windings, or the coils be completely interleaved: first one layer of primary windings, then two secondary, then two primary, etc. Lorch $^{5}$ reported a study in cooperation with Harrowell and others, where winding the superconductor and the protection conductor on separate concentric coils was considered. They concluded that a separate-coil protection technique was not appropriate because of the impossibility of current transfer from one winding to another due to leakage reactances. In fact this is an important problem. mainly for the transfer of current back from an auxiliary winding to a main winding after a fault.

Nevertheless, Harrowell adopted the principle in an extensive study published in $1970^{6}$, in which he added a switch with a parallel resistor to the normally conducting auxiliary winding (Figure $/ b$ ). The resistor had to limit the current in the protection coil under normal circumstances, and was short-circuited by the switch in case of a fault. He suggested that normal resistances would determine the current distribution between main and auxiliary windings during overload. The transformer concept presented by a group at Westinghouse also used auxiliary windings, but without switches. resulting in a completely passive system (Figure lc). The main windings themselves act as switches in case of an overload. In 1982 these workers published experimental data which should prove the validity of this kind of protection system?

Summarizing it can be concluded that the proposed protection techniques have the common feature that a complete switch-off of the transformer is not necessary (that is to say, an immediate switch-off). Protection is achieved by introducing a redundancy in conductor material. either normal or superconductive. which does not contribute to the losses because it is located in a low field region. Only the practical realization has shown any progress (Figure l). 


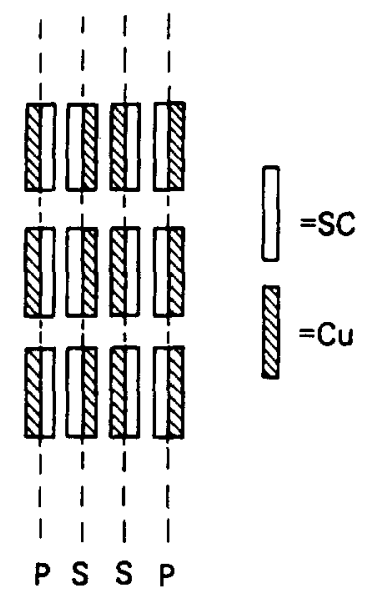

a

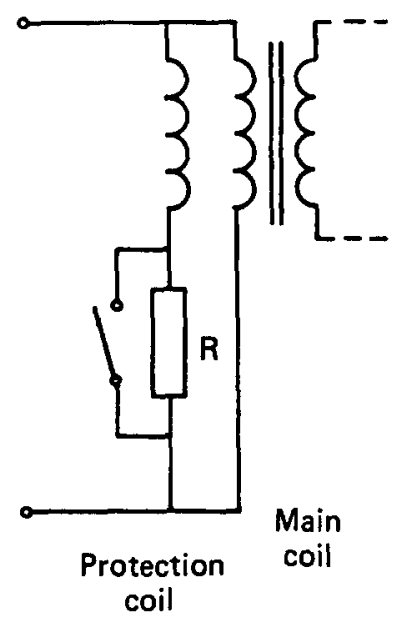

b

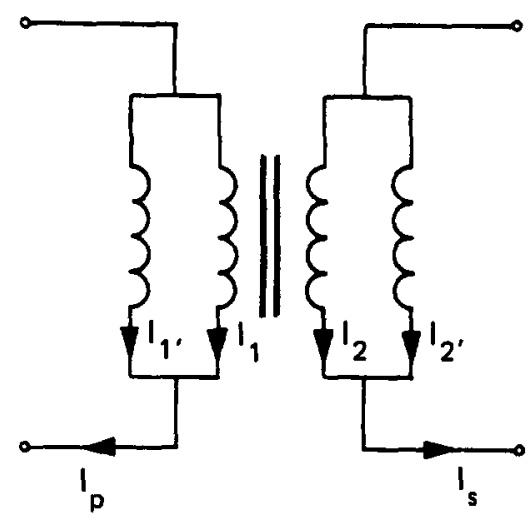

C

Figure 1 Three different protection concepts. a, Suggested by Jones; b, suggested by Harrowell; c, Westinghouse concept discussed in this Paper

As the transformers being considered are to be used mostly as step-up transformers for generators, a protection device must meet the demands that electrical utilities normally make. These include simplicity, extremely reliable operation and the largest possible degree of independence from external power sources. A passive system is therefore more suited to this purpose than any other, and it is the passive double-coil concept which shall be further discussed.

\section{Network representation}

Corresponding main and auxiliary windings have the same number of turns. For the sake of convenience it is assumed here that the primary and secondary coils also have an equal number of turns. This assumption has no qualitative influence on the results compared to those obtained for transformers with a winding ratio unequal to unity.

Coils with an equal number of turns wound around the same high-permeability iron core have self and mutual inductances, $L_{\alpha}, M_{\alpha \beta}$

$$
\begin{aligned}
& L_{\alpha}=L_{0}+\Delta L_{\alpha} \\
& M_{\alpha \beta}=L_{0}+\Delta M_{\alpha \beta}
\end{aligned}
$$

in which

$$
\Delta L_{\alpha} \ll L_{0}, \Delta M_{\alpha \beta} \ll L_{0}
$$

As, for example, the voltage over the resistanceless coil 1

$$
\begin{aligned}
U_{1} & =L_{1} \frac{\mathrm{d} I_{1}}{\mathrm{~d} t}+M_{12} \frac{\mathrm{d} I_{2}}{\mathrm{~d} t}+M_{13} \frac{\mathrm{d} I_{3}}{\mathrm{~d} t}+M_{14} \frac{\mathrm{d} I_{4}}{\mathrm{~d} t} \\
& =L_{0} \frac{\mathrm{d}}{\mathrm{d} t}\left(I_{1}+I_{2}+I_{3}+I_{4}\right)+\Delta L_{1} \frac{\mathrm{d} I_{1}}{\mathrm{~d} t}+\Delta M_{12} \frac{\mathrm{d} I_{2}}{\mathrm{~d} t} \\
& +\Delta M_{13} \frac{\mathrm{d} I_{3}}{\mathrm{~d} t}+\Delta M_{14} \frac{\mathrm{d} I_{4}}{\mathrm{~d} t}
\end{aligned}
$$

should be finite, we can conclude in the limit $L_{0} \rightarrow \infty$, that

$I_{1}+I_{2}+I_{3}+I_{4}=$ constant

and, knowing that the four currents must have initial values of zero

$I_{1}+I_{2}+I_{3}+I_{4}=0$

Connecting two coils oppositely in series (Figure 2), the effective inductance measured at the terminals + and will be defined as the stray inductance between coils $\alpha$ and $\beta$

$$
\begin{aligned}
L_{\sigma \alpha \beta} & =L_{\alpha}+L_{\beta}-2 M_{\alpha \beta} \\
& =\Delta L_{\alpha}+\Delta L_{\beta}-2 \Delta M_{\alpha \beta}
\end{aligned}
$$

$L_{\sigma \alpha \beta}$ has, as a consequence of the second line, a finite value independent of $L_{0}$.

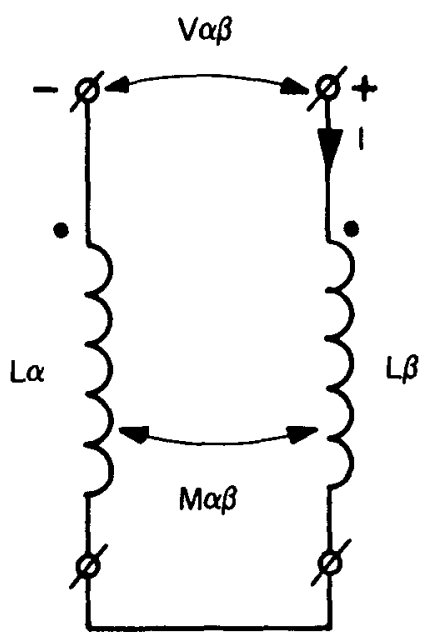

Figure 2 Definition of stray inductance: $V_{\alpha \beta}=L_{\sigma \alpha \beta}(\mathrm{d} / / \mathrm{d} t)$ 
Now applying this to a transformer with two main and two auxiliary windings (Figure $1 c$ ). because all coils have zero resistance. it follows that

$$
\begin{aligned}
U_{1} & =L_{1}^{\prime} \frac{\mathrm{d} I_{1}^{\prime}}{\mathrm{d} t}+M_{1}^{\prime} \frac{\mathrm{d} I_{1}}{\mathrm{~d} t}+M_{1}^{\prime} 2 \frac{\mathrm{d} I_{2}}{\mathrm{~d} t}+M_{1}^{\prime} 2_{2}^{\prime} \frac{\mathrm{d} I_{2}^{\prime}}{\mathrm{d} t} \\
& =M_{1}^{\prime} \frac{\mathrm{d} I_{1}^{\prime}}{\mathrm{d} t}+L_{1} \frac{\mathrm{d} I_{1}}{\mathrm{~d} t}+M_{12} \frac{\mathrm{d} I_{2}}{\mathrm{~d} t}+M_{12}^{\prime} \frac{\mathrm{d} I_{2}^{\prime}}{\mathrm{d} t}
\end{aligned}
$$

for the primary side while an analogous equation holds for the secondary side. Using Equation (6). Equation (7) can be written as

$$
\begin{aligned}
& \left(L_{1}^{\prime}-L_{1}+L_{\sigma_{11}^{\prime}}\right) \frac{\mathrm{d} I_{1}^{\prime}}{\mathrm{d} t}+\left(L_{1}^{\prime}-L_{1}-L_{\sigma_{11}^{\prime}}\right) \frac{\mathrm{d} I_{1}}{\mathrm{~d} t} \\
& +\left(L_{1}^{\prime}-L_{1}-L_{\sigma_{12}^{\prime}}+L_{\sigma_{12}}\right) \frac{\mathrm{d} I_{2}}{\mathrm{~d} t} \\
& +\left(L_{1}^{\prime}-L_{1}-L_{\sigma_{12}^{\prime}{ }^{\prime}}+L_{\sigma_{12}^{\prime}}\right) \frac{\mathrm{d} I_{2}^{\prime}}{\mathrm{d} t}=0
\end{aligned}
$$

which. with Equation (4), yields

$$
\begin{aligned}
& L_{\sigma_{1}^{\prime},}, \frac{\mathrm{d} I_{1}{ }^{\prime}}{\mathrm{d} t}-L_{\sigma_{1}{ }^{\prime},} \frac{\mathrm{d} I_{1}}{\mathrm{~d} t}+\left(L_{\sigma_{12}}-L_{\sigma_{12}{ }^{\prime}}\right) \frac{\mathrm{d} I_{2}}{\mathrm{~d} t} \\
& +\left(L_{\sigma_{12}{ }^{\prime}}-L_{\sigma_{12}{ }^{\prime}}\right) \frac{\mathrm{d} I_{2}^{\prime}}{\mathrm{d} t}=0
\end{aligned}
$$

with its counterpart on the secondary side

$$
\begin{gathered}
\left(L_{\sigma_{12}^{\prime}}-L_{\sigma_{12}^{\prime} \prime^{\prime}}\right) \frac{\mathrm{d} I_{1}^{\prime}}{\mathrm{d} t}+\left(L_{\sigma_{12}}-L_{\sigma_{12}}\right) \frac{\mathrm{d} I_{1}}{\mathrm{~d} t} \\
-L_{\sigma_{22}} \frac{\mathrm{d} I_{2}}{\mathrm{~d} t}+L_{\sigma_{22}}, \frac{\mathrm{d} I_{2}^{\prime}}{\mathrm{d} t}=0
\end{gathered}
$$

The set of Equations (4), (9a) and (9b) completely determines the current distribution in the transformer.

\section{A simple approximation}

A zero order approximation of the different $L_{\sigma \alpha \beta}$ values only takes into account the difference in area of the two circles described by one winding of each of the coils $\alpha$ and $\beta$, respectively. The magnetic energy of $L_{\sigma \alpha \beta}$ is thought to be concentrated in the area between both coils and

$L_{\sigma \alpha \beta}=\mu_{0} \pi n^{2}\left(r_{\mathrm{b}}^{2}-r_{\mathrm{a}}^{2}\right) / l$

Here $n$ is the number of turns of each coil. $l$ is their axial length, $r_{\mathrm{a}}$ is the radius of the inner coil and $r_{\mathrm{b}}$ that of the outer one. This results in

$L_{\sigma \alpha \beta}=L_{\sigma \alpha \gamma}+L_{\sigma \gamma \beta}$ provided that coil $\gamma$ is physically located between coils $\alpha$ and $\beta$. Then Equations (9a) and $(9 b)$ reduce to:

$$
\begin{aligned}
& L_{\sigma_{11}^{\prime}}\left(\frac{\mathrm{d} I_{1}^{\prime}}{\mathrm{d} t}-\frac{\mathrm{d} I_{1}}{\mathrm{~d} t}-\frac{\mathrm{d} I_{2}}{\mathrm{~d} t}-\frac{\mathrm{d} I_{2}{ }^{\prime}}{\mathrm{d} t}\right)=0 \\
& L_{\sigma_{22}{ }^{\prime}}\left(-\frac{\mathrm{d} I_{1}^{\prime}}{\mathrm{d} t}-\frac{\mathrm{d} I_{1}}{\mathrm{~d} t}-\frac{\mathrm{d} I_{2}}{\mathrm{~d} t}-\frac{\mathrm{d} I_{2}{ }^{\prime}}{\mathrm{d} t}\right)=0
\end{aligned}
$$

We then conclude with Equation (4) that

$\frac{\mathrm{d} I_{1}^{\prime}}{\mathrm{d} t}=\frac{\mathrm{d} I_{2}{ }^{\prime}}{\mathrm{d} t}=0$

which. if we have the initial conditions $I_{1}^{\prime}=I_{2}^{\prime}=0$, leads to

$I_{1}^{\prime}=I_{2}^{\prime}=0$

and this is of course the aim because under normal conditions the auxiliary windings are not expected to carry any current. Situations where Equation (13a) is not implied by Equation (13) will become important later in this Paper

\section{A more accurate calculation}

A first order calculation of $L_{\sigma \alpha \beta}$ takes in to account that the projection of a coil on a plane perpendicular to its axis is not a circle but rather a finite area between two concentric circles. When defining the meaning of the radii $r_{2}$ to $r_{5}$ according to Figure 3 , it is found by calculating the magnetic energy from the flux pattern in Figure 3, that

$L_{\sigma \alpha \beta}=\frac{\mu_{0} \pi n^{2}}{6 l}\left(r_{5}^{2}+2 r_{4} r_{5}+3 r_{4}^{2}-3 r_{3}^{2}-2 r_{3} r_{2}-r_{2}^{2}\right)$

Assuming that coil $\beta$ is now a main coil and coil $\alpha$ its auxiliary coil, and the remaining coils are located at radii

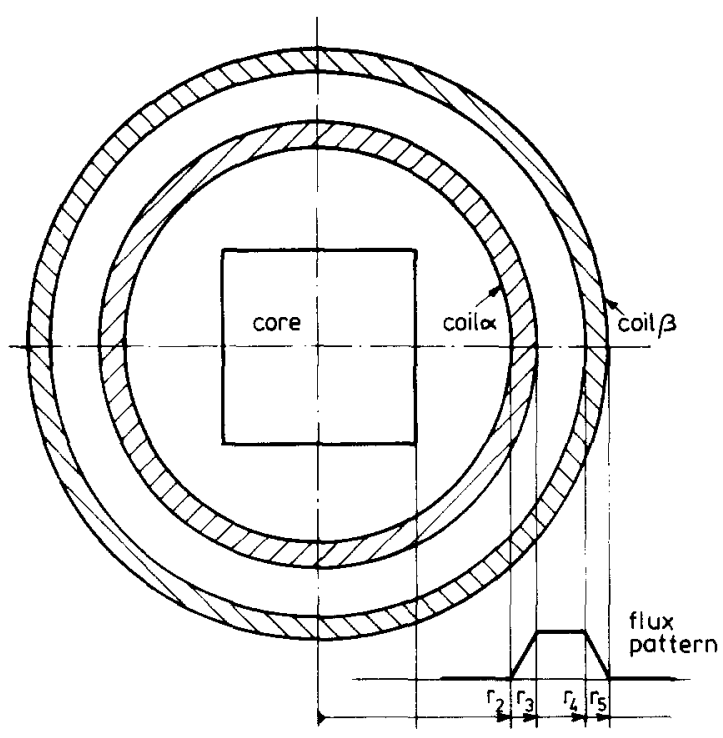

Figure 3 Flux pattern for first order calculation of $L_{\sigma}$ 
$>r_{5}$, then Equations (4), (9a) and (9b) yield

$\frac{\mathrm{d} I_{\alpha}}{\mathrm{d} t} / \frac{\mathrm{d} I_{\beta}}{\mathrm{d} t}=\frac{\left(r_{4}^{2}-r_{5}^{2}\right)}{2 r_{4}^{2}+2 r_{4} r_{5}+2 r_{5}^{2}-3 r_{3}^{2}-2 r_{2} r_{3}-r_{2}^{2}}$

This ratio is always negative, because $r_{2}<r_{3}<r_{4}<r_{5}$. When the differences between the radii are relatively small and $r_{3}-r_{2}=r_{5}-r_{4}=d_{1}, r_{4}-r_{3}=d_{2}$, then

$\frac{\mathrm{d} I_{\alpha}}{\mathrm{d} t} / \frac{\mathrm{d} I_{\beta}}{\mathrm{d} t} \approx-d_{1} /\left(5 d_{1}+6 d_{2}\right)$

\section{Design implications}

For two closely wound coils with comparable dimensions, $I_{1}^{\prime} / I_{1}$ ends up in the range -0.1 to 0.2 . (In a previous publication $^{8}$ a ratio of $1 / 3 \mathrm{~d}_{1} / \mathrm{d}_{2}$ for $\mathrm{d}_{1} \ll \mathrm{d}_{2}$ is suggested; this $1 / 3$ should be $1 / 6$.) So a considerable amount of current flows in the auxiliary winding unless special care is taken in the transformer design to prevent this. That currents in the auxiliary coils should be kept as small as possible is obvious from the fact that these coils consist of normal conductors or largely oversized and wellstabilized superconductors, in which a.c. losses are orders of magnitude higher than in the main conductor, when measured at the same current amplitude.

It can be deduced from Equation (16) that the distance between a main coil and its auxiliary coil must be large compared with the thickness of the main coil. The choice of a very thin main coil is limited both by the amount of superconductive material that is needed and by the fact that for very thin coils, second-order effects illustrated below are becoming more and more important. The option of a very large distance between main and auxiliary coil will have the effect of giving a large $L_{\sigma \alpha \alpha}$ ' value. A high value for this stray inductance puts heavier demands on the recovery characteristics of the main coil, as will be shown below. Modification of the current distribution by giving main and auxiliary coils different numbers of windings is not a solution, because the transformer core flux, which in a technical transformer is a completely independent variable. will then enter into the equations.

Higher order calculations of $L_{\sigma \alpha \beta}$ also include the dependence of the field on the axial direction coordinates. The influence of the latter is comparable to the influence of the transformer iron circuit. This will not be further discussed.

\section{Compensation windings}

It follows from Equations (9a) and (9b) that to obtain $I_{1}^{\prime}=I_{2}^{\prime}=0$, the following must hold

$$
\begin{aligned}
& L_{\sigma_{12}^{\prime}}-L_{\sigma_{12}}-L_{\sigma_{11}^{\prime}}=0 \\
& L_{\sigma_{12}^{\prime}}-L_{\sigma_{12}}-L_{\sigma_{22^{\prime}}}=0
\end{aligned}
$$

This is generally not possible with the configuration of Figure $I c$, but can be accomplished by mounting a small fraction of the auxiliary windings on the other side of the coil set as in Figure 4. Assuming these compensation windings form a very thin coil, and defining $r_{1}$ to $r_{10}$ as in

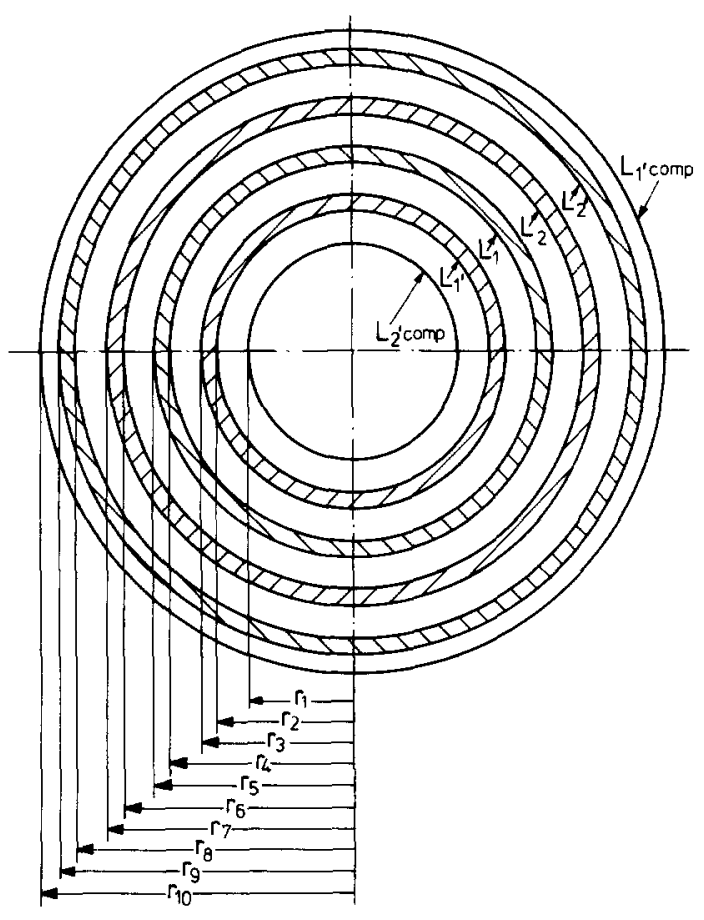

Figure 4 Definition of $r_{1}-r_{10}$ in a six-coil transformer

Figure 4, the first order model introduced above yields, for example for $L_{\sigma_{1}^{\prime} \text {, }}$

$$
\begin{aligned}
L_{\sigma_{1}^{\prime} 1}=\frac{\mu_{0} \pi n^{2}}{6 l} & \times\left[r_{5}^{2}+2 r_{4} r_{5}+3 r_{4}^{2}-3 r_{3}^{2}-2 r_{3} r_{2}-r_{2}^{2}\right. \\
& +2 c_{1}\left(r_{2}^{2}+2 r_{2} r_{3}+3 r_{3}^{2}-2 r_{4}^{2}-2 r_{4} r_{5}-2 r_{5}^{2}\right) \\
& \left.+c_{1}^{2}\left(6 r_{10}^{2}-3 r_{3}^{2}-2 r_{2} r_{3}-r_{2}^{2}\right)\right]
\end{aligned}
$$

Here $c_{1}$ is the part of the windings of $L_{1}^{\prime}$ that has been transferred to the compensation coil.

From Equation (17) it follows that

$c_{1}=1 / 2\left(r_{5}^{2}-r_{4}^{2}\right) /\left(r_{6}^{2}+r_{6} r_{7}+r_{7}^{2}-r_{4}^{2}-r_{4} r_{5}-r_{5}^{2}\right)$

and an analogous equation for a factor $c_{2}$ with $\operatorname{coil} L_{2}{ }^{\prime}$. For relatively small differences in the $r_{i}$

$c_{1} \approx 1 / 6 \frac{\mathrm{d}_{3}}{d_{3}+d_{4}}, r_{5}-r_{4}=r_{7}-r_{6}=d_{3}, r_{6}-r_{5}=d_{4}$

Whether a configuration with this kind of compensation coil is suitable depends on conditions such as the maximum allowable auxiliary-coil current under normal operation and required insulation levels between primary and secondary coils. Realistic figures substituted in Equations (19) or (20) show that only a few percent of the auxiliary-coil windings will have to be transferred, so that a compensation coil will be relatively small. 


\section{Main-coil fault and recovery}

As soon as the critical current of the main coil $L_{\alpha}$ is exceeded a normal spot will develop in the wire. In the arrangement of Figure $I C$ this results in a resistance $R$ in series with the main coil. Recalculating Equations (7) - (9) including $R$, and applying the simplification of Equation (11), we then find that

$L_{\sigma \alpha \alpha^{\prime}} \frac{\mathrm{d} I_{\alpha^{\prime}}}{\mathrm{d} t}=R I_{\alpha}$

A very fast rise of $R$ with time is expected, so that after a negligible time

$R / L_{\sigma \alpha \alpha^{\prime}} \gg \omega$

in which $\omega$ is the a.c. power frequency. For this expectation to be correct the conductor needs a good heat conductivity in its axial direction, as well as a normal resistance for the complete coil obeying expression (22). Formula (22) does not hold for a conventional power transformer, but for one with superconducting windings it will even when the windings have some kind of copper stabilization. If expression (22) is true, it can be assumed that the total current $I_{10 t}=I_{\alpha}+I_{\alpha}$, is constant for the time needed to allow $L_{\alpha}$ to become completely normal. For this time interval Equation (21) can be written as

$L_{\sigma \alpha \alpha^{\prime}} \frac{\mathrm{d} I_{\alpha}}{\mathrm{d} t}+R I_{\alpha}=0$

where $R$ is a function of $I_{\alpha}$ and of time. Multiplication by $l_{\alpha}$ yields

$1 / 2 L_{\sigma \alpha \alpha^{\prime}} I_{\alpha}^{2}+\int R I_{\alpha}^{2} \mathrm{~d} t=$ constant

$I_{\alpha}$ will approach zero, whereby energy equal to $1 / 2 L_{\sigma \alpha \alpha^{\prime}} I_{\alpha}{ }^{2}$ is dissipated in coil $L_{\alpha}$. It must be able to cope with this energy adiabatically. As coils are at present manufactured with the same amount of superconductive material, but with inductances orders of magnitude higher, which are able to dissipate all of their stored energy in their windings, this should not be a serious problem.

A stationary situation will develop where $I_{\alpha}$ is determined by the total current $I_{\text {tor }}$, the magnitude of the inverse power frequency $1 / \omega$ as compared with the thermal time constants of $L_{\alpha}$, or, when these time constants are all relatively small (favourable), by its $V-I$ curve after a quench.

These quantities also determine the current at which $L_{\alpha}$ will again become fully superconducting. A superconductor which recovers at a high effective value of $I_{\mathrm{tot}}$ is necessary to realize a transformer with self-recovering windings. Mixed matrix conductors are expected to be the most promising solution.

\section{After recovery}

Recovery of a complete superconducting state in $L_{\alpha}$ will probably occur at the point in time when $I_{\alpha}=0$. i.e. when $\mathrm{d} I_{\alpha} / \mathrm{d} t=0$. At this moment $I_{\alpha}$, has an extreme value, which means that $L_{\alpha}$ from this time on will carry a small a.c. current superimposed upon this extreme, which from now on will become the amplitude of the a.c. component of $I_{\alpha}$.
As $I_{\text {tot }}$ has no d.c. component. $I_{\alpha}$ has a d.c. component opposite to that of $I_{\alpha^{\prime}}$; in other words, $I_{\alpha}$ varies between zero and twice the value $I_{\text {tot }}$ has at the moment of recovery. The d.c. component will fall to zero according to the time constant determined by $L_{\sigma \alpha \alpha}$, and the total normal resistance in the closed loop $L_{\alpha}-L_{\alpha}$. When the auxiliary coil is also made of superconducting wire. this time constant could reach an unwanted high value. Deliberately constructing or introducing a normal spot in the auxiliary winding will be enough to create an acceptable time constant. In any case, if the main winding has to recover at any value of the current below the nominal current of the transformer, it should be able to carry at least twice this current.

\section{Experiments}

In the low-loss conductor test facility of the Twente University of Technology, loss measurements have been performed in a transformer configuration with main windings protected in the way reviewed above. Results of the loss measurements will be published elsewhere?. In this Paper the behaviour of the protection system is the subject of discussion.

Four coils of 240 windings each were wound on a glass-fibre cylinder, according to Figure 5 . Both main coils were made of a low-loss high-resistivity matrix composite without copper stabilization. The auxiliary windings were wound using a copper-stabilized multifilamentary wire Between every two layers of windings a layer of axially directed thin copper wires was laid to conduct dissipated heat to the liquid helium bath. The complete set of coils was then vacuum impregnated with Stycast 2850 resin (Figure 6). Figure 5 shows the dimensions of the coils. The stray inductances were measured and found to vary between $\approx 90\left(L_{\sigma_{12}}\right)$ and $360 \mu H\left(L_{\sigma_{12}^{\prime}}\right)$. Unfortunately it has not been possible to measure these inductances with the accuracy necessary to verify the current distribution theory. The theoretical values according to (14) are 89 and $558 \mu H$. respectively. The differences are due to the simplicity of Equation (14) and to the fact that the radii of the coils are not very accurately known.

By means of cryotrons. as shown in Figure 7 . it is possible to switch off any of the four coil currents. The normal resistance of each switch amounts to $\approx 10 \Omega$

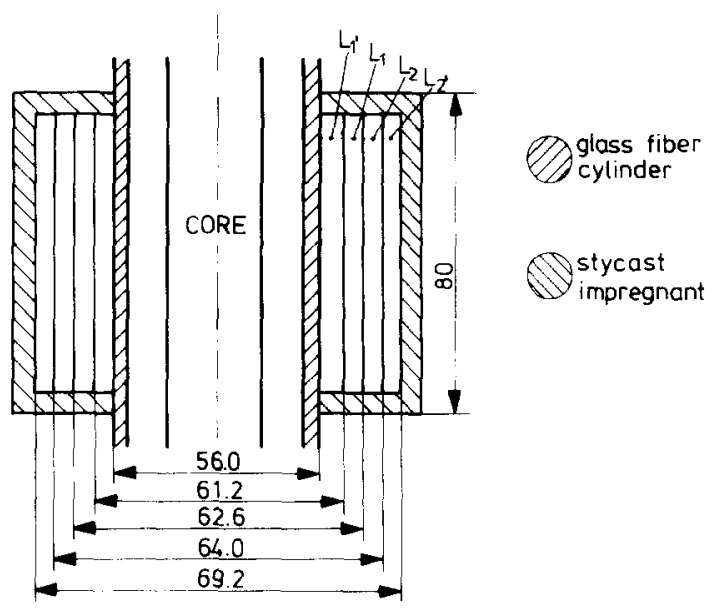

Figure 5 Dimensions of experimental transformer coil set (in $\mathrm{mm}$ ) 


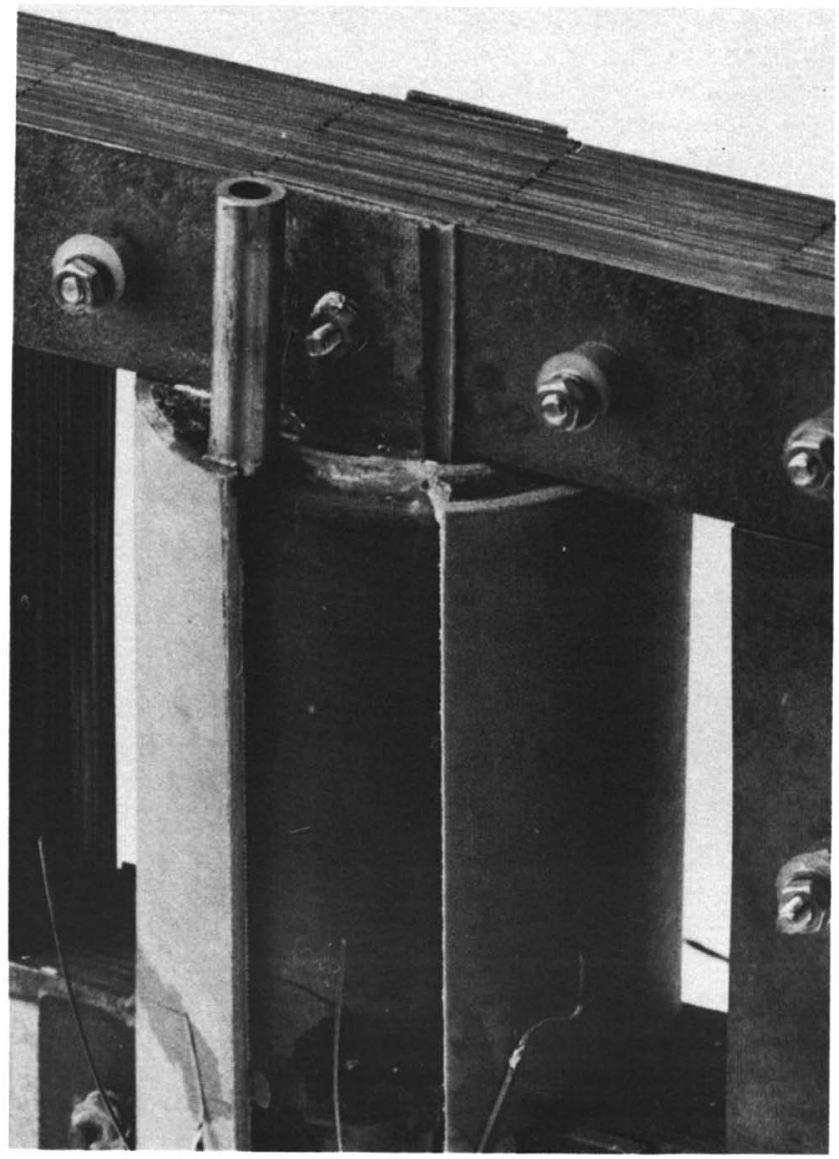

Figure 6 Photograph of the transformer. The coil set was mounted in a closed housing to permit calorimetric loss measurements

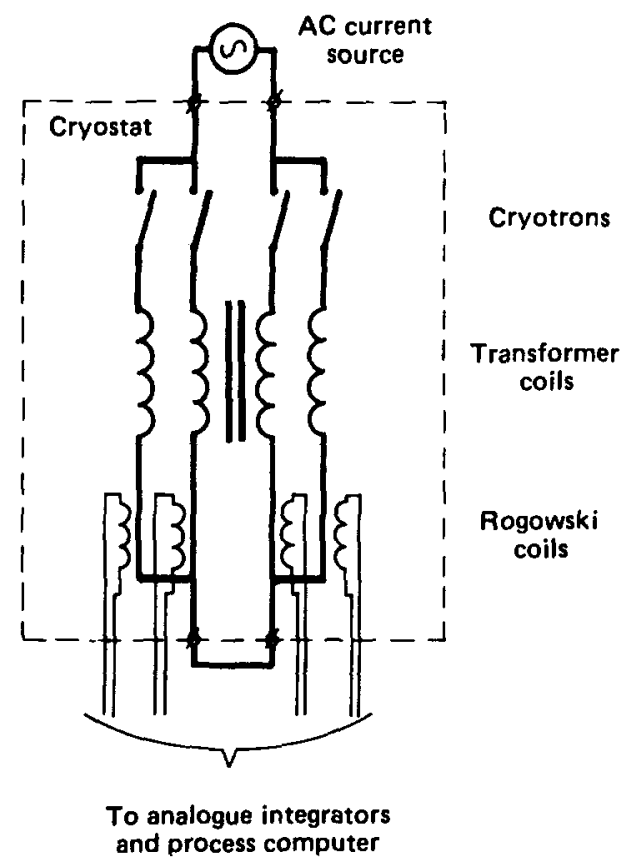

Figure 7 Set-up of transformer, switches and Rogowski coils

Apart from this, heater elements were later mounted on one of the leads of $L_{1}^{\prime}$ and on one of $L_{2}{ }^{\prime}$. By means of these heaters a normal spot of several tens of micro-ohms can be introduced in one of the terminals of an auxiliary coil.

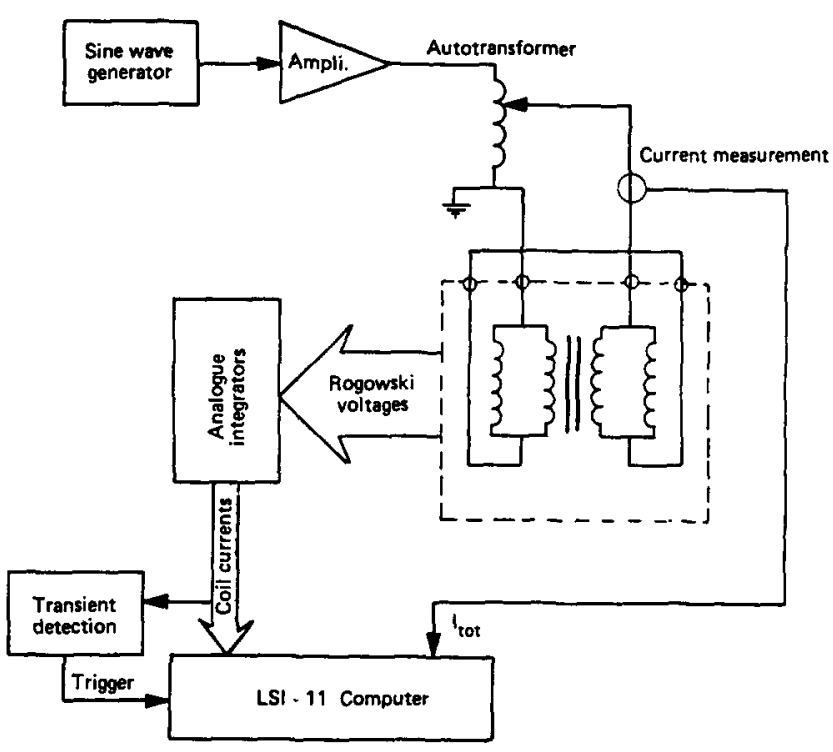

Figure 8 Measurement set-up

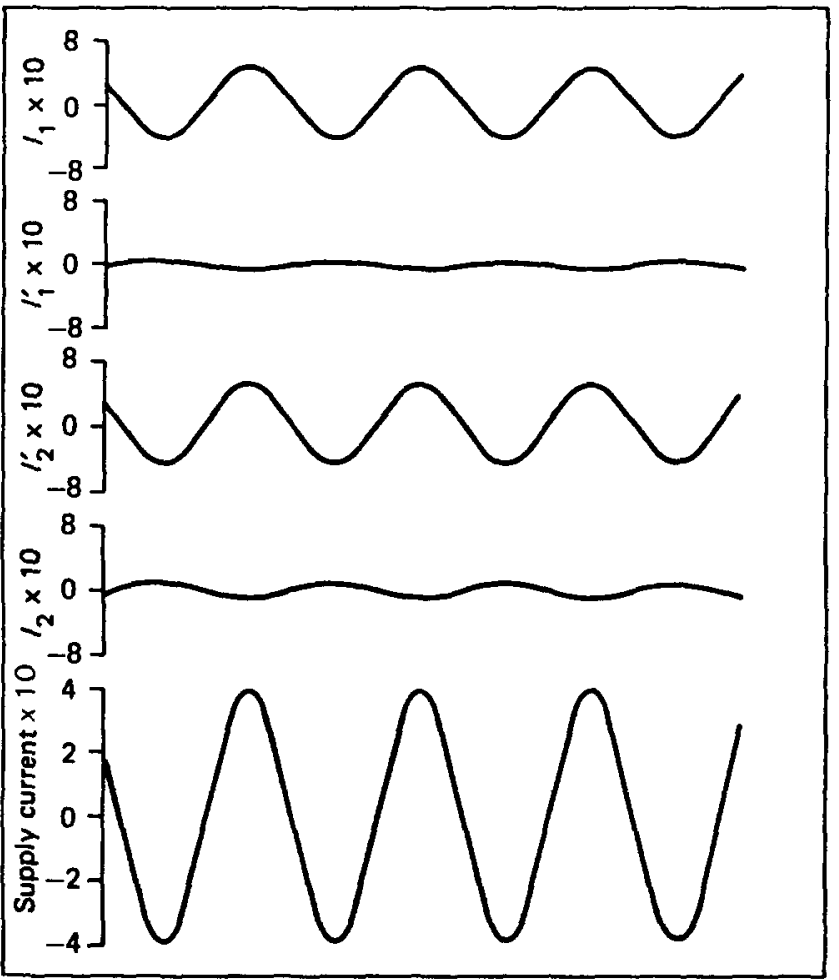

Figure 9 Current distribution and transients: normal operation, coil currents $80 \mathrm{~A}$ per division. Supply currents, $I_{2}, I_{2}^{\prime}, I_{1}^{\prime}$ and $I_{1}$ are plotted from bottom to top as a function of time. Signal frequency $40 \mathrm{~Hz}$

By means of Rogowski coils, the four coil currents can be measured separately (apart from d.c. currents). The integrators used have a high-pass cut-off time constant of $\approx 6 \mathrm{~s}$.

Operational behaviour under normal circumstances has been measured in the experimenal set-up shown in Figure 8 . The sample frequency of the process computer was $2000 \mathrm{~Hz}$ for each signal and the signal frequency was $40 \mathrm{~Hz}$. The resulting current distribution is shown in Figure 9. Switching off a main winding by means of its 


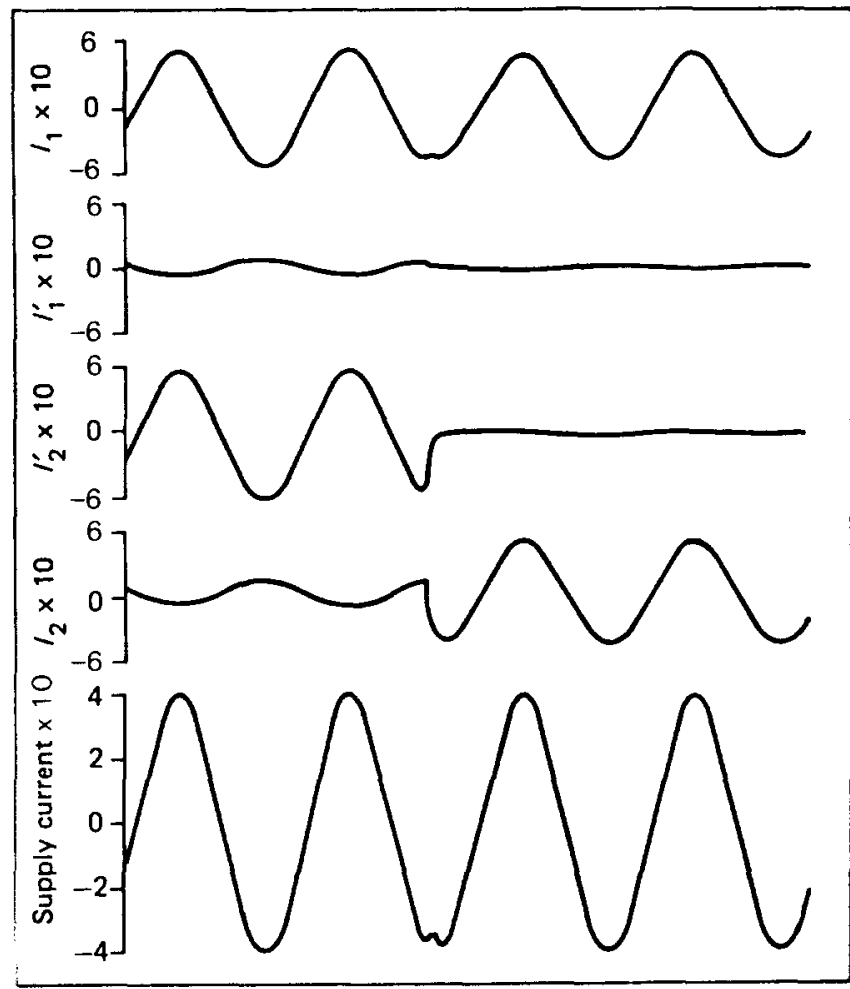

Figure 10 Current distribution and transients: $L_{2}$ switched off, coil currents 60 A per division. Supply currents, $I_{2}, I_{2}^{\prime}, I_{1}^{\prime}$ and $I_{1}$ are plotted from bottom to top as a function of time. Signal frequency $40 \mathrm{~Hz}$

cryotron influences the current distribution in the other pair of coils as well. as is illustrated by Figures 10 and $I 1$. Figure 12 shows the behaviour of the currents when a quench occurs in the transformer itself, due to exceeding the maximum current of the wire.

To record quench events, the computer was triggered by the strongly changing inductive voltage occuring at a quench. The quench was stimulated by slowly increasing the amplitude of the a.c. current. As can be seen, the current commutates to the other coil in a very short time. A large number of quench experiments did not affect the properties of the main coil conductors, proving this kind of protection to be very effective and reliable.

It is possible that only one of both coils quenches. but as the primary and secondary windings are exactly alike, quenching of the one almost always instantaneously triggers quenching of the other. Even switching off one of the main coils at a somewhat lower current (40 A whereas the quench current is $50 \mathrm{~A}$ peak value). triggers quenching of the other coil. The current values mentioned here relate to the current fed from the power supply into the cryostat. Due to the reverse currents in the auxiliary windings, the main winding currents are higher.

Recovery of the main coils occurs at currents between 0.5 and $2 \mathrm{~A}$ peak value (non-reproducing) at $f=40 \mathrm{~Hz}$. At $f=10 \mathrm{~Hz}$ and lower. higher values are found. which are only reproducible when the amplitude of the current source is decreased very slowly and smoothly. Figure 13 gives a good example of the recovery phenomenon at $f=5 \mathrm{~Hz}$. Triggering of the computer was performed by detecting an increased a.c. amplitude in $L_{1}$. The current in $L^{\prime}$ was switched off manually after recovery was observed. The figure clearly shows a persistent d.c. current being switched off.

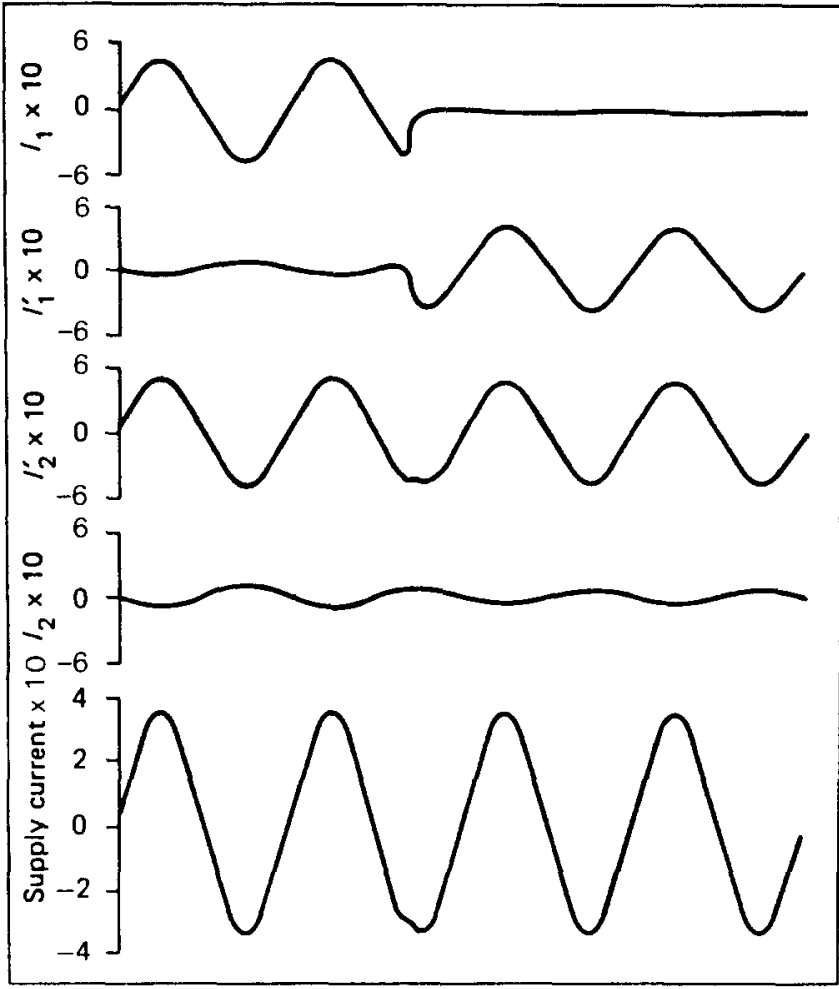

Figure 11 Current distribution and transients: $L_{1}$ switched off, coil currents 60 A per division. Supply currents, $I_{2}, I_{2}^{\prime}, I_{1}^{\prime}$ and $I_{1}$ are plotted from bottom to top as a function of time. Signal frequency $40 \mathrm{~Hz}$

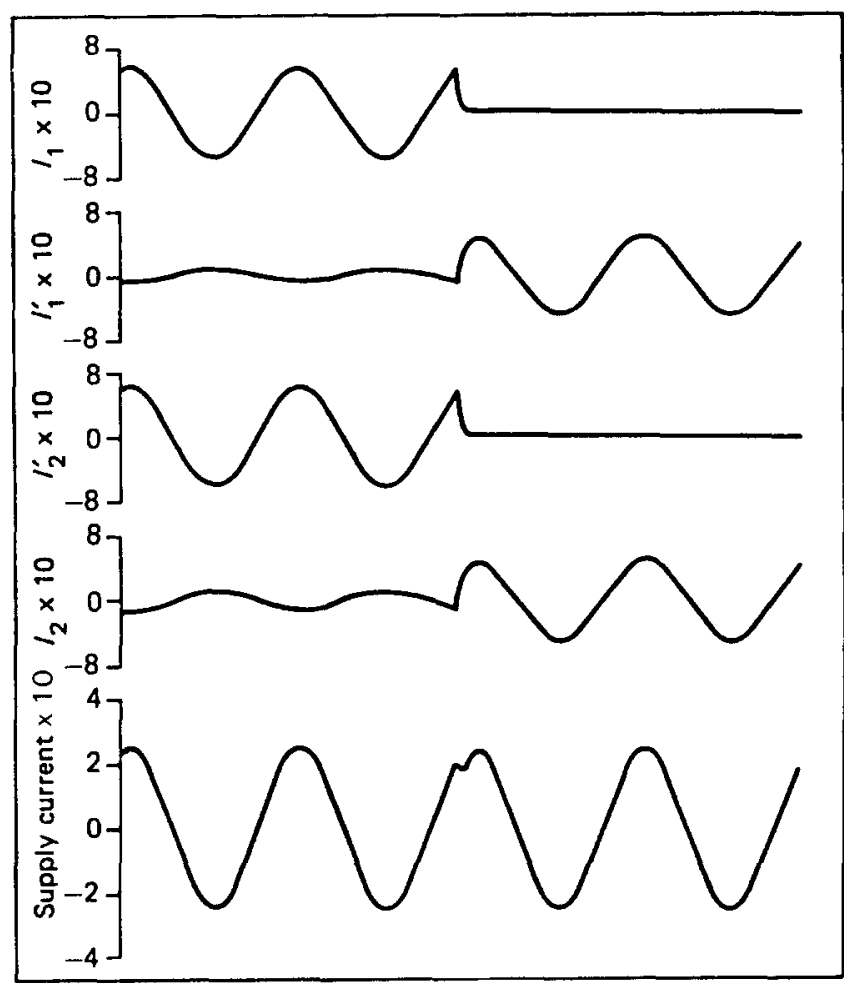

Figure 12 Current distribution and transients: quench by slowly increasing current amplitude, coil currents $80 \mathrm{~A}$ per division. Supply current $I_{2}, I_{2}^{\prime}, I^{\prime}$ and $I$, are plotted from bottom to top as a function of time. Signal frequency $40 \mathrm{~Hz}$

Figure 14, presenting measurements at $f=8 \mathrm{~Hz}$. shows the coil currents as a function of time when the heaters in the terminals of both auxiliary coils are 


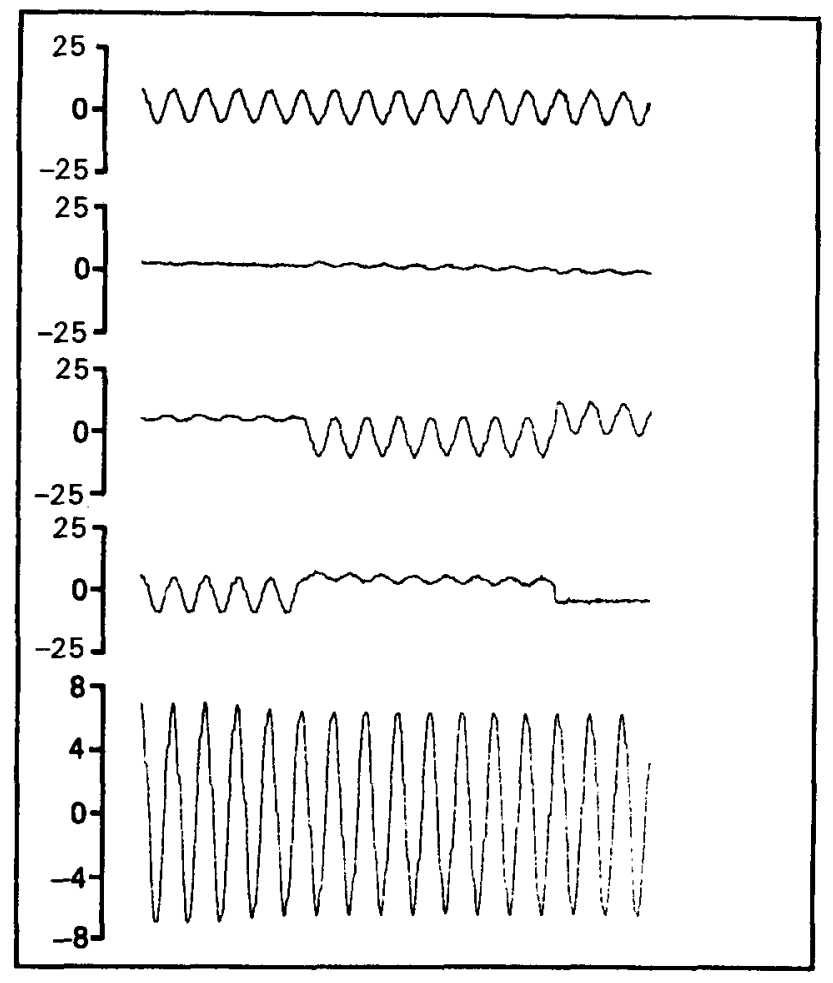

Figure 13 Current distribution and transients: recovery of $L_{2}$, and $L_{2}{ }^{\prime}$ switched off, coil currents $25 \mathrm{~A}$ per division. Supply currents, $I_{2}, I_{2}^{\prime}, I_{1}^{\prime}$ and $I_{1}$ are plotted from bottom to top as a function of time. Signal frequency $5 \mathrm{~Hz}$

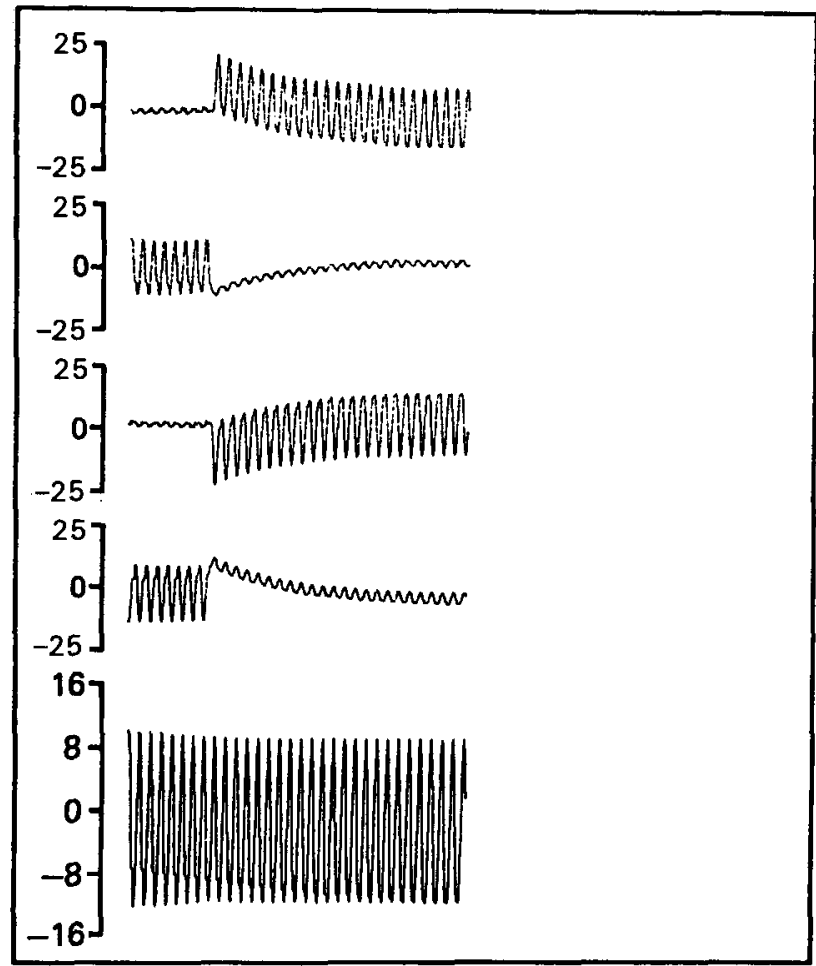

Figure 14 Current distribution and transients: recovery of main coils with heated coil terminals, coil currents $25 \mathrm{~A}$ per division. Supply currents, $I_{2}, I_{2}^{\prime}, I_{1}^{\prime}$ and $I_{1}$ are plotted from bottom to top as a function of time. Signal frequency $8 \mathrm{~Hz}$

activated. Now the d.c. component of the current behaves transiently and is damped within a few seconds.

\section{Discussion}

The current distribution experiment (Figure 9) shows that the coil design of the transformer is far from optimal. Calculations using Equation (14) predict a ratio $I_{i \mathrm{a}} I_{\mathrm{a}}^{\prime} \approx-6$ for the primary as well as the secondary side, whereas the measured values are $I_{1} / I_{1}^{\prime}=-8.0$ and $I_{2} / I_{2}^{\prime}=-5.4$.

Beside this, Figures 10 and $I 1$ show that the current distribution in one pair of coils influences that in the other pair. This influence cannot be shown from the results of Equation (14). The reasons that higher order effects play such an important role are mainly that the main coils are extremely thin and that there are no gaps between the different coils.

As to the problem of current transfer. it is especially clear from Figure 12 that a very fast transfer from main to auxiliary coil is possible. Some experimental work will be needed to verify if this also applies to main coils with mixed matrix conductors. Transfer of current back to a main coil has proved to be impossible in the experimental transformer at a normal power frequency. The better performance expected of a mixed matrix conductor will have also to be investigated.

It can be seen from, for example. Figure 13 that before recovery a certain amount of current already flows in the main coil, which is a strongly non-sinusoidal function of time. This proves there is a rapid growth and decrease of the normal spot(s) in the conductor. In the case of a mixed matrix conductor, this current will be much higher and will have to be taken into consideration in Equations (21)-(24). Theoretical work should reveal in which way most profit can be obtained from such non-linear behaviour.

Finally, it will have to be investigated if it would be better not to have a completely superconducting auxiliary coil (the advantages and disadvantages of a $\mathrm{Cu}$ or $\mathrm{Al}$ coil at $77 \mathrm{~K}$ will not be discussed here), to prevent the occurrence of long-time d.c. transients after fault recovery. The use of switches to dissipate d.c. transients is an unnecessary complication of the system.

\section{Conclusions}

The validity of a protection technique for superconducting transform windings with no active parts has been demonstrated. The current in the auxiliary windings under normal circumstances can be kept very low, when the auxiliary windings are partly located on the other side of the coil set. The recovery behaviour of a wire with only a high resistivity matrix is very poor. The conductor probably needs a better longitudinal conductivity to improve this.

\section{Acknowledgements}

This work was supported by the Netherlands Foundation for Fundamental Research on Matter (FOM) and by SMIT NYMEGEN Superconducting Magnet Systems, Nijmegen. The Netherlands.

\section{References}

l Riemersma, H., Riemersma, H., Barton, M.L., Eckels, P.W., Litz, D.C., Murphy, J.H., Roach, J.F. IEEE Trans on Power Apparatus and Systems PAS-100 (1981) 10, 3398-3405

2 Dubots, P., Dubois, P., Fevrier, A., Renard, J.C., Goyer, J., Ky, H.G. ICEC 10 Otaniemi. Finland (1984) 610

3 Dubots, P., Février, A., Renard, J.C., Tavergnier, J.P., Goyer, J., Ky, H.G. IEEE Trans Magnet (1985) MAG-21 177

4 Jones, J.E. Proc IEE (1964) 111 1195-1198

5 Lorch, H.O. Cryogenics (1969) $9354-36$ )

6 Harrowelh, R.V. Proc IEE (1970) 117 133-140)

7 Murphy, J.H. Adv Cryogenic Eng (1982) 27 73-80

8 van Overbeeke, F., Oordt, K., Van de Klundert, L.J.M. ICEC IO Otaniemi, Finland (1984) 715

9 van Overbeeke, F., Oordt K. and van de Klundert, L.J.M. IEEE Trans Magnet (1985) MAG-21 366 